\title{
Magnetic resonance white matter changes in patients with obstructive sleep apnoea. The subset of the PURE-MIND (Prospective Urban and Rural Epidemiological) cohort study
}

Katarzyna Postrzech-Adamczyk ${ }^{1,2}$, Artur Nahorecki², Jagoda Jacków-Nowicka3 , Maria Wołyniec ${ }^{4}$, Maciej Karczewski ${ }^{5}$, Robert Skomro ${ }^{2,6}$, Anna Zimny ${ }^{3}$, Andrzej Szuba²

${ }^{1}$ Department of Internal Medicine, $4^{\text {th }}$ Military Hospital, Wroclaw, Poland ²Department of Angiology, Hypertension and Diabetology, Wroclaw Medical University, Wroclaw, Poland

${ }^{3}$ Department of General and Interventional Radiology and Neuroradiology, Wroclaw Medical University, Wroclaw, Poland

${ }^{4}$ Department of Social Medicine, Wroclaw Medical University, Wroclaw, Poland ${ }^{5}$ Department of Mathematics, Faculty of Environmental Engineering and Geodesy, Wrocław University of Environmental and Life Sciences, Wroclaw, Poland ${ }^{6}$ University of Saskatchewan, Saskatoon, Canada

Submitted: 4 March 2020

Accepted: 14 July 2020

Arch Med Sci

DOI: https://doi.org/10.5114/aoms.2020.100676

Copyright (c) 2020 Termedia \& Banach

\section{Abstract}

Introduction: Changes typical for cerebral small vessel disease (i.a. white matter hyperintensities - WMHs) are often found accidentally in neuroimaging studies. Although asymptomatic, this condition increases the risk of future ischaemic incidents and neurodegenerative disorders. Sleep apnoea is a recognised risk factor for vascular diseases. The aim of our study was to assess the prevalence of, and association between, obstructive sleep apnea (OSA) and cerebral small vessel disease in the studied population.

Material and methods: Two hundred and seven patients (participants of Prospective Urban Rural Epidemiology Study) took part in our study. The study group consisted of 31 patients with OSA ( 11 women and 20 men). Nine of them were diagnosed with mild OSA, nine with moderate OSA, and 13 with severe OSA. The control group consisted of 176 patients (133 women and 43 men) who scored 0-2 points in the STOP-BANG questionnaire. All patients underwent brain magnetic resonance imaging. We evaluated the occurrence and severity of WMHs.

Results: Significantly higher incidence of WMHs was found in the study group compared to controls. In univariate analyses, age was a significant predictor of periventricular white matter changes. For subcortical area, age and waist-to-hip ratio were significant predictors.

Conclusions: Significantly higher incidence of WMHs in the studied group suggests that patients with OSA may have a higher risk of neurodegeneration.

Key words: obstructive sleep apnoea, cerebral small vessel disease, magnetic resonance imaging, white matter hyperintensities.

\section{Introduction}

Cerebral circulation disorders are one of the main causes of mortality and permanent disability. According to American data, when considered
Corresponding author: Katarzyna PostrzechAdamczyk Department of Internal Medicine $4^{\text {th }}$ Military Hospital 5 Rudolfa Weigla St 53-114 Wroclaw, Poland E-mail: kpostrzech. adamczyk@gmail.com 
separately from other cardiovascular diseases, stroke ranks fifth among all causes of death, behind diseases of the heart, cancer, chronic lower respiratory tract disease, and unintentional injuries [1]. Cerebral small vessel disease (CSVD) refers to a group of pathological processes with various aetiologies, which affect the small arteries, arterioles, venules, and capillaries of the brain. The consequences of CSVD on the brain parenchyma can be detected in neuroimaging studies and consist mainly of lesions located in the subcortical structures such as lacunar infarcts, white matter lesions, large haemorrhages, and microbleeds. Clinical implications of CSVD are as follows: increased risk of ischaemic stroke, disability, and cognitive decline in the elderly. Because this constitutes an important and - as we can anticipate in connection with the aging of society - growing problem, attention should be directed to modifiable risk factors. The link between hypertension, obesity, hyperglycaemia, hyperlipidaemia, renal dysfunction, behavioural factors (smoking, sedentary lifestyle, unhealthy diet), and CSVD is recognised and documented [2, 3]. Another significant factor associated with stroke risk is obstructive sleep apnoea (OSA). Obstructive sleep apnoea is characterised as the occurrence of apnoeas or hypopnoeas during sleep. The polysomnography-determined Apnoea-Hypopnoea Index (AHI) defines the severity of the disease. ApnoeaHypopnoea Index between $5-15 / \mathrm{h}$ indicates mild OSA, whereas $15-30 / \mathrm{h}$ points to moderate OSA and $>30 / \mathrm{h}$ to severe OSA. It is a common disorder affecting millions of people. Data from the Wisconsin Cohort Study estimate the overall prevalence of OSA at $33.9 \%$ in men and $17.4 \%$ in women between 30 and 70 years of age, while moderate to severe OSA (defined by $\mathrm{AHI}>15 / \mathrm{h}$ ) affects $13.0 \%$ of men and $5.6 \%$ of women [4]. Large analyses indicate that severe OSA is associated with a doubling of stroke risk, higher post-stroke mortality, and worse functional outcome $[5,6]$. The aim of our study was to assess the prevalence and association between CSVD and OSA in Polish subjects taking part in the Prospective Urban Rural Epidemiology (PURE) study, which is a population cohort study of individuals from urban and rural communities from 21 countries.

\section{Material and methods}

The PURE study is a large prospective cohort study with the main objective of investigating the association between societal influences on human lifestyle, cardiovascular risk factors, and the rate of noncommunicable diseases [7]. PURE MIND (a sub-study of the PURE study) was designed to determine the risk factors and consequences of silent cerebrovascular disease detected on brain magnetic resonance imaging (MRI). Participants were interviewed using a standardised questionnaire, completed medical examination, and the STOP-BANG questionnaire, and they underwent brain MRI. Data included biometrics, cardiovascular disease risk factors, health history, use of medications, and body composition analysis. Ethical approval was acquired from the Local Ethical Committee. All participants signed an informed consent form.

\section{Study population}

Two hundred and seven subjects with no history of cerebrovascular disease were included in the study. They were participants of PURE MIND sub-study, who filled in the STOP-BANG questionnaire. A total of 176 patients (133 women and 43 men) scored 0-2 and were categorised as the control group. The remaining group of 31 who scored > 3 (11 women and 20 men) underwent full-night polysomnography and were diagnosed with OSA using the AASM Manual for the Scoring of Sleep and Associated Events, version 2.5. All patients had brain MRI. Body composition analysis was performed using a BC-554 IRONMAN ${ }^{\circledR}$ Body Composition Monitor (Tanita). The demographic characteristics of the study population are presented in Table I. Hypertension was defined as systolic blood pressure over $140 \mathrm{~mm} \mathrm{Hg}$ or diastolic blood pressure over $90 \mathrm{~mm} \mathrm{Hg}$ measured at the time of visit as well as treatment with antihypertensive drugs. Diabetes was defined either as treatment with antidiabetic drugs or fasting glucose over $7 \mathrm{mmol} / \mathrm{l}(126 \mathrm{mg} / \mathrm{dl})$ (measured twice) or glucose over $>11.1 \mathrm{mmol} / \mathrm{l}(140 \mathrm{mg} / \mathrm{dl})$ after two hours in oral glucose tolerance test. Hyperlipidaemia was diagnosed if serum total cholesterol level was over $12.2 \mathrm{mmol} / \mathrm{l}(220 \mathrm{mg} / \mathrm{dl})$ or if the patient was undergoing treatment with antihyperlipidemic medications.

\section{Sleep apnoea evaluation}

The STOP-BANG questionnaire was used as a screening tool for OSA. It includes eight dichotomous (yes/no) questions related to the clinical features of sleep apnoea (Snoring, Tiredness, Observed apnoea, high blood Pressure, BMI, Age, Neck circumference, and male Gender). The total score ranges from 0 to 8 . Patients can be classified for their OSA risk based on their respective scores. It has been evaluated as a three-category variable (low risk (0-2), medium risk (3-4), high risk (5-8)) and validated in pre-operative and general populations [8-10]. The available data demonstrate that a STOP-Bang score $<2$ excludes moderate and severe OSA with a probability of $95 \%$; therefore, this was used in our study $[11,12]$. The low 
Magnetic resonance white matter changes in patients with obstructive sleep apnoea. The subset of the PURE-MIND (Prospective Urban and Rural Epidemiological) cohort study

Table I. Characteristics of the studied population

\begin{tabular}{|c|c|c|c|}
\hline Parameter & OSA group & Control group & P-value \\
\hline Male, $n(\%)$ & $20(64.52)$ & $43(24.43)$ & 0.0000254 \\
\hline Female, $n(\%)$ & $11(35.48)$ & $133(75.57)$ & \\
\hline Age [years] & $63.06 \pm 6.55$ & $55.64 \pm 7.73$ & 0.000004 \\
\hline Body mass index $\left[\mathrm{kg} / \mathrm{m}^{2}\right]$ & $31.52 \pm 4.66$ & $25.57 \pm 3.84$ & 0.000000001 \\
\hline Waist-hip ratio & $0.9341 \pm 0.07$ & $0.8400 \pm 0.08$ & 0.00000003 \\
\hline Hypertension, $n$ (\%) & $24(77.42)$ & $45(25.57)$ & $5.79 \mathrm{e}-8$ \\
\hline Diabetes, $n(\%)$ & $9(29.03)$ & $8(4.55)$ & 0.000129 \\
\hline $\begin{array}{l}\text { Elevated serum total } \\
\text { cholesterol [mg/dl] }\end{array}$ & $19(61.29)$ & $71(40.34)$ & 0.0481 \\
\hline $\mathrm{HDL}[\mathrm{mg} / \mathrm{dl}]$ & $55.97 \pm 14.45$ & $65.00 \pm 15.16$ & 0.002 \\
\hline LDL [mg/dl] & $119.7 \pm 35.22$ & $126.4 \pm 36.19$ & 0.4 \\
\hline TG [mg/dl] & $135.0 \pm 75.89$ & $115.7 \pm 59.95$ & 0.1 \\
\hline $\mathrm{HbA}_{1 \mathrm{c}}(\%)$ & $5.845 \pm 0.82$ & $6.151 \pm 10.57$ & 0.00008 \\
\hline Taking aspirin, $n(\%)$ & $6(19.36)$ & $10(5.68)$ & 0.0186 \\
\hline Current smoking, $n(\%)$ & $3(9.68)$ & $29(16.48)$ & 0.00000687 \\
\hline Smoking in the past, $n$ (\%) & $22(70.97)$ & $45(25.57)$ & \\
\hline Body fat (\%) & $34.39 \pm 8.413$ & $30.88 \pm 8.476$ & 0.07 \\
\hline Body water (\%) & $46.36 \pm 5.194$ & $50.64 \pm 38.395$ & 0.4 \\
\hline Muscle mass (\%) & $56.24 \pm 10.02$ & $45.53 \pm 8.58$ & 0.000004 \\
\hline
\end{tabular}

Data expressed as mean \pm standard deviation. OSA - obstructive sleep apnoea, HDL - high-density lipoprotein, LDL - low-density lipoprotein, TG - triglycerides. Conversion factors to SI units are as follows: for total cholesterol, HDL and LDL 0.02586; for TG 0.0113.

OSA risk group was considered as control group. Other participants underwent full in-lab diagnostic night polysomnography (Embletta ${ }^{\circledR}$ MPR Sleep System with RemLogic software). All polysomnography studies were scored by a physician blinded to the results of MRI. Apnoea was defined as a cessation of airflow for at least 10 seconds. Hypopnoea was defined as an amplitude reduction of airflow that was associated with an oxygen desaturation of $>3 \%$ or an arousal. Obstructive sleep apnoea was defined by $\mathrm{AHI}>5 / \mathrm{h}$. Patients with $\mathrm{AHI} 5-15 / \mathrm{h}$ were diagnosed with mild OSA, AHI 15-30/h - moderate OSA, and AHI > 15/h severe OSA.

\section{Brain magnetic resonance imaging evaluation}

All patients underwent MR of the brain. The data were acquired using a 1.5 T MR (GE) scanner and 16-channel HNS coil. Imaging protocol consisted of T1, T2, FLAIR, and SWI images. The presence of ischaemic stroke, changes in white matter with high signal on T2-weighted and FLAIR images (WMHs - white matter hyperintensities), and micro- or macrobleeds were assessed by trained radiologists. Because no stroke was found and the amount of micro- and macrobleeds was statistically insignificant, only WMHs were taken for further analysis. The severity of WHMs in periventricular and subcortical areas was evaluated using the Fazekas scale [13]. This scoring system includes four grades depending on the size and confluence of lesions: grade 0 - absent, grade 1 - punctate, grade 2 - early confluent, grade 3 - confluent.

\section{Statistical analysis}

All statistical analyses were performed using $R$ for windows statistical software (version 3.6.1). The results were expressed as the mean \pm standard deviation. Mann-Whitney test and Kruskal-Wallis test were used to compare continuous variables when appropriate. Fisher exact test was performed for qualitative variables. Correlation between continuous variables was assessed using Spearman correlation coefficient. A $p$-value less than 0.05 was considered statistically significant.

\section{Results}

Among 31 OSA subjects 13 (4 women and 9 men) had severe OSA, 9 (4 women and 5 men) 
Table II. Polysomnography results

\begin{tabular}{|l|c|c|c|}
\hline \multirow{2}{*}{ Sex } & \multicolumn{3}{|c|}{ OSA } \\
\cline { 2 - 4 } & Mild (AHI 5-15/h) & Moderate (AHI 15-30/h) & $\begin{array}{c}\text { Severe (AHI > 30/h) } \\
(n=13)\end{array}$ \\
\hline \multirow{2}{*}{ Female $(n=11)$} & Mean AHI: $9.57 \pm 3.31$ & Mean AHI: $19.40 \pm 3.41$ & Mean AHI: $62.15 \pm 16.42$ \\
& $n=3$ & $n=4$ & $n=4$ \\
\hline \multirow{2}{*}{ Male $(n=20)$} & Mean AHI: $10.03 \pm 3.36$ & Mean AHI: $21.72 \pm 3.81$ & Mean AHI: $53.90 \pm 21.05$ \\
& $n=6$ & $n=5$ & $n=9$ \\
\hline
\end{tabular}

Data expressed as mean \pm standard deviation. OSA - obstructive sleep apnoea, AHI - Apnoea-Hypopnoea Index.

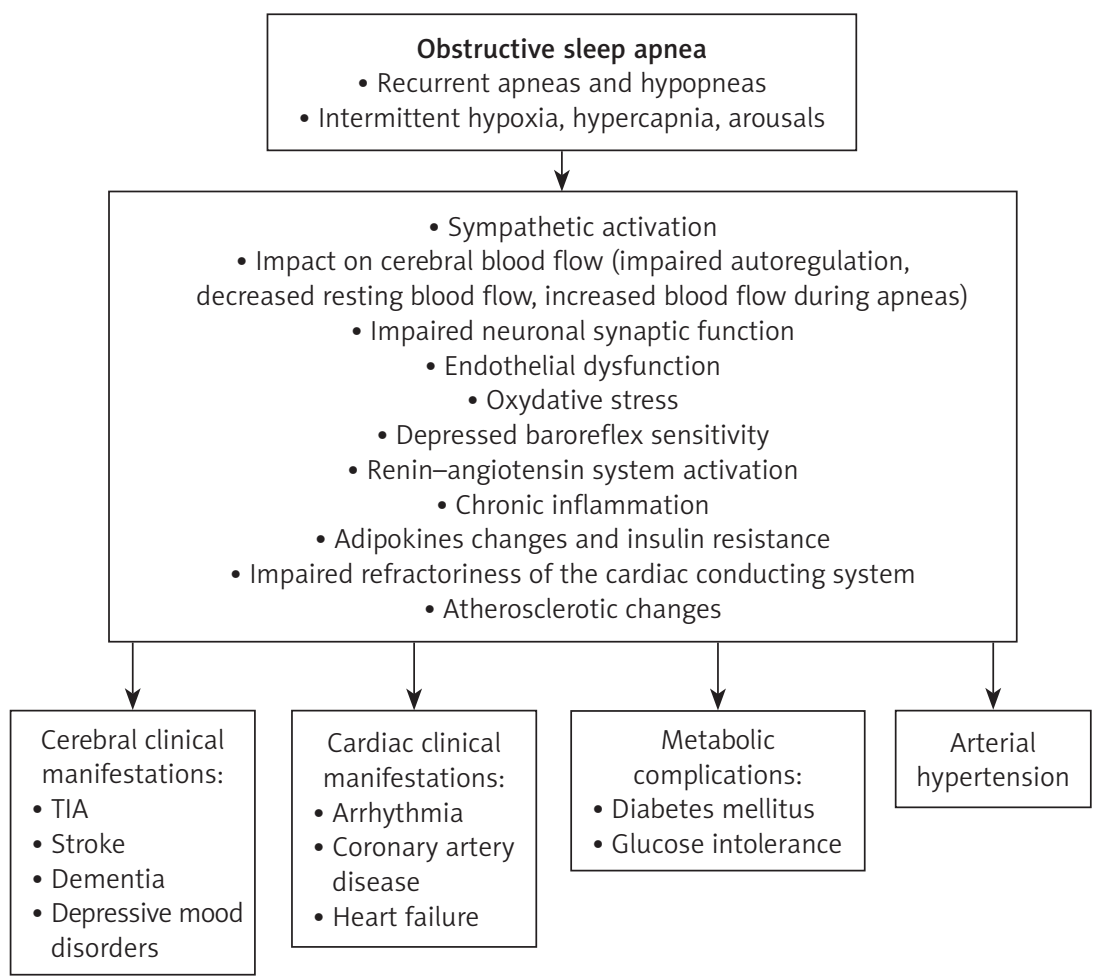

Figure 1. Pathophysiological mechanisms and complications of obstructive sleep apnoea

were diagnosed with moderate OSA, and the other 9 ( 3 women and 6 men) had mild OSA. Mean AHI in female and male groups was similar $(32.26 \pm 25.78$ vs. $32.70 \pm 24.50, p=0.9$ ). Results of PSG are listed in Table II. Magnetic resonance imaging showed WMHs in periventricular area in $10(32.25 \%)$ subjects in the OSA group and 19 (10.79\%) subjects in the control group, and the difference was statistically significant $(p=0.00382)$. The prevalence of subcortical lesions was also higher in OSA patients $(n=13 ; 41.94 \%)$ than in the control group ( $n=29 ; 16.48 \% ; p=0.00580)$ No major ischaemic strokes, lacunar strokes, or microbleeds were revealed. In univariate analyses we used the prevalence of WMHs as a dependent variable and assessed the impact of the following independent variables: age, gender, BMI, WHR, smoking, hypertension, hyperglycaemia, hyperlipidaemia, use of aspirin, body muscle mass, body water, and body fat. Among these independent variables only age
( $p=0.002$ or $p=0.01$ ) was a predictor of the prevalence of periventricular WMHs in patients with OSA and control subjects. For subcortical WMHs age (both in the OSA and control group; $p=0.0002$ or $p=0.04$ ) and WHR (only in OSA group $p=0.03$ ) were significant predictors. On the other hand, we used $\mathrm{AHI}$ as a dependent variable in the OSA group and evaluated the impact of the same independent variables and WMHs, but no significant predictor was identified in this group.

\section{Discussion}

Aging and neurodegenerative disorders are a growing problem. White matter hyperintensities along with perivascular space widening, brain atrophy, and lacunar infarctions are neuroradiological manifestations of CVSD and are histopathologically correlated with axonal loss, focal myelinolysis, reactive astrocytosis, and vessel wall hyalinosis. Clinical implications include 
increased risk of stroke, cognitive dysfunction, dementia, gait instability, and depression [14, 15]. Typically, changes increase with the aging process; however, their onset and progression can be exacerbated by comorbidities. The prevalence of CSVD in neuroimaging studies ranges from $5 \%$ to $62 \%$ [16], depending on the studied population and research methodology. In most papers this incidence was estimated at 10-20\%. Higher values are observed in patients with previous stroke or transient ischaemic attack (TIA). The strongest factor influencing the occurrence is age, with an annual incidence of between $2 \%$ and $4 \%$. In the Rotterdam Scan Study, the prevalence of silent brain infarcts increased from $8 \%$ in subjects 60 to 64 years old, $13 \%$ in 65 to 69 years old, to more than $20 \%$ in the 70 to 79 -year-olds and $35 \%$ in those older than 80 years [17]. As well as age, other significant risk factors that have a strong association with CSVD are hypertension, hyperlipidaemia, smoking, male sex, and intra- and extracranial large artery atherosclerotic disease [18-20]. The effects of other recognised cardiovascular factors such as diabetes mellitus, tobacco use, alcohol consumption, obesity, or atrial fibrillation, although possibly important, have not been clearly demonstrated. One of the suggested factors contributing to the onset and severity of CSVD is OSA. Pathophysiological changes that occur during periods of apnoea and hypopnoea affect the vascular endothelium and flow in the cerebral vessels both at night and during wakefulness [21]. Oxidative stress, inflammatory response, activation of the sympathetic nervous system, and disturbed blood flow autoregulation interfere with normal blood flow in the cerebral macro- and microvessels. Numerous studies have demonstrated that patients with moderate and severe OSA have a higher prevalence of CSVD than controls or subjects with mild OSA [22-26]. A recently published meta-analysis and systematic reviews confirm that moderate to severe sleep apnoea is independently related to $\mathrm{WMH}$ and silent brain infarcts, which strongly suggests that OSA may contribute to the pathogenesis of CSVD $[27,28]$. In the present study the score of WMHs both in periventricular and subcortical areas was significantly higher in patients with OSA than in the control group. Our analysis indicated age as the strongest risk factor for WMHs, consistent with previous findings. Major strengths of our study include the population representative group [29], recruitment strategies to mitigate bias, and no previous studies on the population in this region. There are, however, some limitations of our study: the small sample size, the lack of exclusion of OSA on the basis of polysomnography, age differences, and gender asymmetry favouring men in the OSA group and women in the control group, which limits the external validity of the results. In future research the comparison with a control group of similar age, sex, and comorbidities could determine the relationship between OSA and CSVD more clearly. Some data suggest that effective hypertension control can prevent or halt WMH progression [30-32]. Although it remains unproven whether continuous positive pressure treatment (CPAP) also has such an effect, some reports indicate that CPAP may positively alter brain microstructure and perfusion patterns [33]. The study of Castronovo et al. showed that the structural brain abnormalities observed in white matter regions susceptible to hypoxemia can improve with CPAP treatment in three months. The effect was more pronounced after 12 months of positive pressure treatment [34]. In another study assessing not only resonance image but also cognitive function tests, significant improvement in the verbal episodic memory test was seen after just one month of using CPAP [35]. Further investigations evaluating the long-term effect of OSA treatment on brain microarchitecture on large groups of patients are needed.

In conclusion, the significantly higher incidence of WMHs in the OSA group suggests that patients with OSA may be at increased risk of neurodegenerative complications.

\section{Acknowledgments}

Partially funded by grants 290/WPURE/2008/0 from the Polish Ministry of Science and Higher Education and 2015/17/B/NZ7/02963 from the Polish National Science Centre. The PURE-Poland study also was partially funded by the Population Health Research Institute in Hamilton, Canada.

\section{Conflict of interest}

The authors declare no conflict of interest.

\section{References}

1. Benjamin EJ, Muntner P, Alonso A, et al. Heart disease and stroke statistics - 2019 update: a report from the American Heart Association. Circulation 2019; 139: e56-e528.

2. Pantoni L. Cerebral small vessel disease: from pathogenesis and clinical characteristics to therapeutic challenges. Lancet Neurol 2010; 9: 689-701.

3. Assallum H, Song TY, Aronow W, Chandy D. Obstructive sleep apnoea and cardiovascular disease: a literature review. Arch Med Sci 2019. DOI: https://doi.org/10.5114/ aoms.2019.88558.

4. Peppard PE, Young T, Barnet JH, et al. Increased prevalence of sleep-disordered breathing in adults. Am J Epidemiol 2013; 177: 1006-14.

5. Parra O, Arboix A, Montserrat JM, et al. Sleep-related breathing disorders: impact on mortality of cerebrovascular disease. Eur Respir J 2004; 24: 267-72.

6. Xie C, Zhu R, Tian Y, Wang K. Association of obstructive sleep apnoea with the risk of vascular outcomes and 
all-cause mortality: a meta-analysis. BMJ Open 2017; 7 : 013983.

7. Teo K, Chow CK, Vaz M, Rangarajan S, Yusuf S; PURE Investigators-Writing Group. The Prospective Urban Rural Epidemiology (PURE) study: examining the impact of societal influences on chronic noncommunicable diseases in low-, middle-, and high-income countries. Am Heart J 2009; 158: 1-7.e1.

8. Nagappa M, Wong J, Singh M, Wong DT, Chung F. An update on the various practical applications of the STOP Bang questionnaire in anesthesia, surgery, and perioperative medicine. Curr Opin Anaesthesiol 2017; 30: 118-25.

9. Chung F, Abdullah HR, Liao P. STOP-Bang questionnaire. Chest 2016; 149: 631-8.

10. Chung F, Yegneswaran B, Liao P, et al. STOP questionnaire: a tool to screen patients for obstructive sleep apnea. Anesthesiology 2008; 108: 812-21.

11. Christensson E, Franklin KA, Sahlin C, et al. Can STOP bang and pulse oximetry detect and exclude obstructive sleep apnea? Anesth Analg 2018; 127: 736-743.

12. Kuczyński W, Mokros Ł, Stolarz A, Białasiewicz P. The utility of STOP-BANG questionnaire in the sleep-lab setting. Sci Rep 2019; 9: 6676.

13. Fazekas F, Chawluk JB, Alavi A. MR signal abnormalities at $1.5 \mathrm{~T}$ in Alzheimer's dementia and normal aging. Am J Neuroradiol 1987; 8: 421-6.

14. Lei C, Deng Q, Li H, Zhong L. Association between silent brain infarcts and cognitive function: a systematic review and meta-analysis. J Stroke Cerebrovasc Dis 2019; 28: 2376-87.

15. Vermeer SE, Longstreth WT, Koudstaal PJ. Silent brain infarcts: a systematic review. Lancet Neurol 2007; 6: 611-9.

16. Fanning JP, Wong AA, Fraser JF. The epidemiology of silent brain infarction: a systematic review of population-based cohorts. BMC Med 2014; 12: 119.

17. Vermeer SE, Koudstaal PJ, Oudkerk M, Hofman A, Breteler MMB. Prevalence and risk factors of silent brain infarcts in the population-based Rotterdam scan study. Stroke 2002; 33: 21-5.

18. De Havenon A, Meyer C, Mcnally JS, Alexander M, Chung L. Subclinical cerebrovascular disease: epidemiology and treatment. Curr Atheroscler Rep 2019; 21: 39.

19. Prabhakaran S, Wright CB, Yoshita M, et al. The prevalence and determinants of subclinical brain infarction: the Northern Manhattan Study. Neurology 2008; 70: 425-30.

20. Nam KW, Kwon HM, Jeong HY, et al. Cerebral white matter hyperintensity is associated with intracrania atherosclerosis in a healthy population. Atherosclerosis 2017; 265: 179-83.

21. Hoffmann M, Wolf J, Szyndler A, Singh P, Somers VK, Narkiewicz K. Serum of obstructive sleep apnea patients impairs human coronary endothelial cell migration. Arch Med Sci 2017; 13: 223-7.

22. Nishibayashi M, Miyamoto M, Miyamoto T, Suzuki K, Hirata K. Correlation between severity of obstructive sleep apnea and prevalence of silent cerebrovascular lesions. J Clin Sleep Med 2008; 15: 242-7.

23. Koo DL, Kim JY, Lim JS, Kwon HM, Nam H. Cerebral microbleeds on MRI in patients with obstructive sleep apnea. J Clin Sleep Med 2017; 13: 65-72.

24. Song TJ, Park JH, Choi K, et al. Moderate-to-severe obstructive sleep apnea is associated with cerebral small vessel disease. Sleep Med 2017; 30: 36-42.

25. Del Brutto OH, Mera RM, Zambrano M, Castillo PR. Relationship between obstructive sleep apnea and neu- roimaging signatures of cerebral small vessel disease in community-dwelling older adults. The Atahualpa Project. Sleep Med 2017; 37: 10-2.

26. Lee $\mathrm{MH}$, Yun $\mathrm{CH}, \mathrm{Min} \mathrm{A}$, et al. Altered structural brain network resulting from white matter injury in obstructive sleep apnea. Sleep 2019; 42: zsz120.

27. Huang Y, Yang C, Yuan R, Liu M, Hao Z. Association of obstructive sleep apnea and cerebral small vesse disease: a systematic review and meta-analysis. Sleep 2020; 43: zsz264.

28. Ho BL, Tseng PT, Lai CL, et al. Obstructive sleep apnea and cerebral white matter change: a systematic review and meta-analysis. J Neurol 2018; 265: 1643-53.

29. Corsi DJ, Subramanian SV, Chow CK, et al. Prospective Urban Rural Epidemiology (PURE) study: baseline characteristics of the household sample and comparative analyses with national data in 17 countries. Am Heart J 2013; 166: 636-46.

30. Dufouil C, Chalmers J, Coskun O, et al. Effects of blood pressure lowering on cerebral white matter hyperintensities in patients with stroke: The PROGRESS (Perindopril Protection Against Recurrent Stroke Study) Magnetic Resonance Imaging Substudy. Circulation 2005; 112 : 1644-50.

31. De Havenon A, Majersik JJ, Tirschwell DL, et al. Blood pressure, glycemic control, and white matter hyperintensity progression in type 2 diabetics. Neurology 2019; 92: 1168-75.

32. Godin O, Tzourio C, Maillard P, Mazoyer B, Dufouil C. Antihypertensive treatment and change in blood pressure are associated with the progression of white matter lesion volumes: the Three-City (3C)-Dijon Magnetic Resonance Imaging Study. Circulation 2011; 123: 266-73.

33. Maresky HS, Shpirer I, Klar MM, et al. Continuous positive airway pressure alters brain microstructure and perfusion patterns in patients with obstructive sleep apnea. Sleep Med 2019; 57: 61-9.

34. Castronovo V, Scifo P, Castellano A, et al. White matter integrity in obstructive sleep apnea before and after treatment. Sleep 2014; 37: 1465-75.

35. Rosenzweig I, Glasser M, Crum WR, et al. Changes in neurocognitive architecture in patients with obstructive sleep apnea treated with continuous positive airway pressure. EBioMedicine 2016; 7: 221-9. 